\title{
Transforming AHP Group Decisions into Fuzzy Priorities - A New Methodology to Aggregate Individual Priorities
}

\author{
Oliver Meixner*, Sonja Hackl and Rainer Haas \\ Institute of Marketing and Innovation \\ University of Natural Resources and Life Sciences Vienna, Austria \\ * Corresponding author: oliver.meixner@boku.ac.at
}

\begin{abstract}
The following contribution suggests a new and to our knowledge previously unpublished approach to combine AHP group judgements considering the possible variation of individual AHP evaluations. By introduction fuzzy logic into the group decision process, this approach is appropriate to cover the distribution of individual judgements. The paper will present in brief the theoretical foundation of our approach. Afterwards, we will use an empirical example to test the methodology. For this purpose, we use data of a previously conducted survey, where an expert group evaluated the sustainability of palm oil by means of a comprehensive AHP model.
\end{abstract}

Keywords: group decision making, fuzzy AHP, expert knowledge, sustainability.

\section{Introduction}

AHP group decision making (GDM) is a widely used methodology to solve decision problems not only by an individual evaluation but by a number of usually well-informed decision makers. If the group of decision makers is working together as a unit, a usual approach is to aggregate individual judgments (AIJ). However, quite often expert judgments depend on various individual positions. In this case, aggregation of individual priorities (AIP) are appropriate (Forman and Peniwati, 1998). In this contribution, we show how individual priorities that were approximated by applying the Analytic Hierarchy Process (AHP) (Forman and Peniwati, 1998) can be aggregated by means of the Fuzzy Analytic Hierarchy Process (FAHP) theory. Crisp numbers are transformed into fuzzy numbers. By this approach - and this will be shown on a concrete, real world example - it is possible to show the whole range of opinions in cases where consensus may hardly be reached. The research question of this contribution is: It is possible to aggregate individual AHP priorities in a non-consensus decision situation by transferring crisp AHP priorities into fuzzy AHP priorities? This could be relevant in particular in decision situations where the result of a group evaluation process does not necessarily lead to the selection of one specific alternative but should rather show the whole range of opinions.

\section{Literature Review}

A number of publications are dealing with the question of reaching a consensus within a group of decision makers (Van Den Honert, 1998; Dong et al., 2010; Srdjevic et al., 2013). However, for specific goals it might be even advantageous to cover the whole spectrum of 
answers. In this case, the following contribution is proposing a new approach of covering different opinions of decision making by transforming individual priorities into fuzzy numbers. This approach differs from existing ones, like the Aggregation of Individual Preference Structures (AIPS) (Escobar and Moreno-Jiménez, 2007), that individual objectives of different actors are completely or at least partly incorporated into the approximation of an aggregated preference structure.

Fuzzy logic can be traced back Zadeh's fuzzy sets and number of the mid 1960ies, first published in 1965 (Zadeh, 1965) and was also introduced into the AHP theory. Examples of the application of the Fuzzy AHP are found in natural resources management such as water management (Srdjevic and Medeiros, 2008), for industrial applications such as satellite mobile communication systems (Ling and $\mathrm{Wu}, 2004$ ) or computer integrated manufacturing systems (Bozdag, Kahraman and Ruan, 2003), in project management and team formation (Wi et al., 2009), to name just a few. There is also a limited body of knowledge available covering, e.g., the application of fuzzy hierarchy multiple attributes to deal with imprecision of expert judgments (Chang, Wu and Lin, 2009) (authors applied AIJ in combination of FAHP). However, to our knowledge, the full potential of FAHP was not used to cover decision situations where a consensus can hardly be reached amongst decision makers and is even not strived.

\section{Objectives}

Within our study we will show that the proposed methodology of transforming individual evaluations using the AHP (crisp numbers) into fuzzy priorities will help to better understand the whole range of evaluations in a group decision situation. This is in particular relevant where a compromise or consensus can hardly be reached, which is usually due to differing opinions, expertise, and positions of the parties involved. The approach can be used for any AHP group decision and is not depending on any other pre-conditions than the usual ones (AHP axioms (Saaty, 1995)). The methodology will therefore rather lead to a new interpretation of heterogeneous group evaluations than proposing new theoretical inputs to AHP. All basic foundations are already existing and broadly discussed in literature. Therefore, we will refrain from formulating well-known theoretical foundations and focus on the concrete application of the new aggregation procedure.

\section{Methodology}

When introducing fuzzy logic into the AHP, the following assumptions have to be considered. Usually, fuzzy logic was integrated into AHP theory by using triangular fuzzy numbers. Hereby, the membership function $\mu(x)$ of a triangular fuzzy number $\widetilde{M}=$ $(l, m, u)$ with a lower limit $l$, a medium value $m$ and an upper limit $u$ can be described as (Chang, 1996, 650):

$\mu(x)=\left\{\begin{array}{cl}\frac{x-l}{m-l}, & x \in[l, m] \\ \frac{u-x}{u-m}, & x \in[m, u] \\ 0, \text { otherwise }\end{array}\right.$

It is therefore possible - with a membership function $\mu(x)$ reaching from 0 to 1 - that $\widetilde{M}_{1}$ amounts from $l$ to $u$, whereby $\mu(x)=1$ for $m$ and $\mu(x)=0$ for all values that are smaller than $l$ and larger than $u . \widetilde{M}_{1}$ is therefore covering a whole spectrum of possible outcomes 
and not only one data point when crisp numbers are used. The basic operations of triangular fuzzy numbers $\widetilde{M}_{1}=(l, m, u)$ number $\widetilde{M}_{2}=(l, m, u)$ are:

$\widetilde{M}_{1} \oplus \widetilde{M}_{2}=\left(l_{1}+l_{2}, m_{1}+m_{2}, u_{1}+u_{2}\right)$

$\widetilde{M}_{1} \otimes \widetilde{M}_{2} \approx\left(l_{1} l_{2}, m_{1} m_{2}, u_{1} u_{2}\right)$

$\widetilde{M}_{1}^{-1} \approx \frac{1}{u_{1}}, \frac{1}{m_{1}}, \frac{1}{l_{1}}$

Suppose we have $K$ decision makers evaluating an AHP decision hierarchy containing $I$ elements. Consequently, we get a priority vector $W_{i k}, i=1 \ldots I, k=1 \ldots \mathrm{K}$. The basic idea of our approach-using AIP without eliminating the range of approximations - is to aggregate the individual crisp priorities $W_{i k}$ into one fuzzy priority vector

$\widetilde{W}_{i k}=\left\{\min \left(W_{i k}\right), \bar{W}_{i k}, \max \left(W_{i k}\right)\right\}$.

The upper limit $l$ represents the minimum of all priorities for element $i$, the medium $m$ for element $i$ is calculated by the average priority (arithmetic mean), and the upper limit $u$ is represented by the maximum of all priorities for element $i$. If the decision hierarchy is exhibits more than one hierarchy level, the multiplicative aggregation of weightings has to be done by means of the basic fuzzy operations above (Formula 2 to 4). Consequently, we approximate fuzzy weights instead of highly aggregated crisp numbers covering the whole spectrum of evaluations in a group decision situation. The following numerical example visualizes this approach.

\section{Data/Model Analysis}

The data used in the following example were collected within a recently finished and unpublished study where decision makers, a panel of experts in their field, evaluated the sustainability of palm oil. The AHP is widely used in natural resources management including GDM, e.g., water management (Srdjevic, 2007; Calizaya et al., 2010), bioenergy (Buchholz et al., 2009), or land management (Cay and Uyan, 2013). The applied AHP hierarchy was elaborated on the basis of a comprehensive literature review. The evaluation of the alternatives (see below) was done by means of quantitative information taken from previous studies. Consequently, the main task of the experts was to evaluate the importance of the criteria of the decision hierarchy by means of pairwise comparisons. The top level of the sustainability criteria consisted of the usual elements environmental, economic, and social criteria, going back to the so-called Brundtland Report of 1987 (Kuhlman and Farrington, 2010). Below this level, each of these elements was further structured into 2 to 5 sub-criteria containing measurable sustainability characteristics (e.g., "climate change" as one important element of ecological sustainability, measured in $\mathrm{CO}_{2}$-equivalents of a given alternative). The following table shows the whole decision hierarchy and also contains the approximation of the alternatives "conventional palm oil" (non-certified), "RSPO-certified palm oil", and "rapeseed oil" (as a reference product). The metric numbers are mainly based on literature covering, e.g., life cycle assessment of crops (Schmidt, 2010). 
ISAHP Article: A Style Guide for Paper Proposals To Be Submitted to the International Symposium on the Analytic Hierarchy Process 2020, Web Conference.

Table 1. Criteria and alternatives of the decision hierarchy to evaluate the sustainability of palm oil incl. metric values and weights.

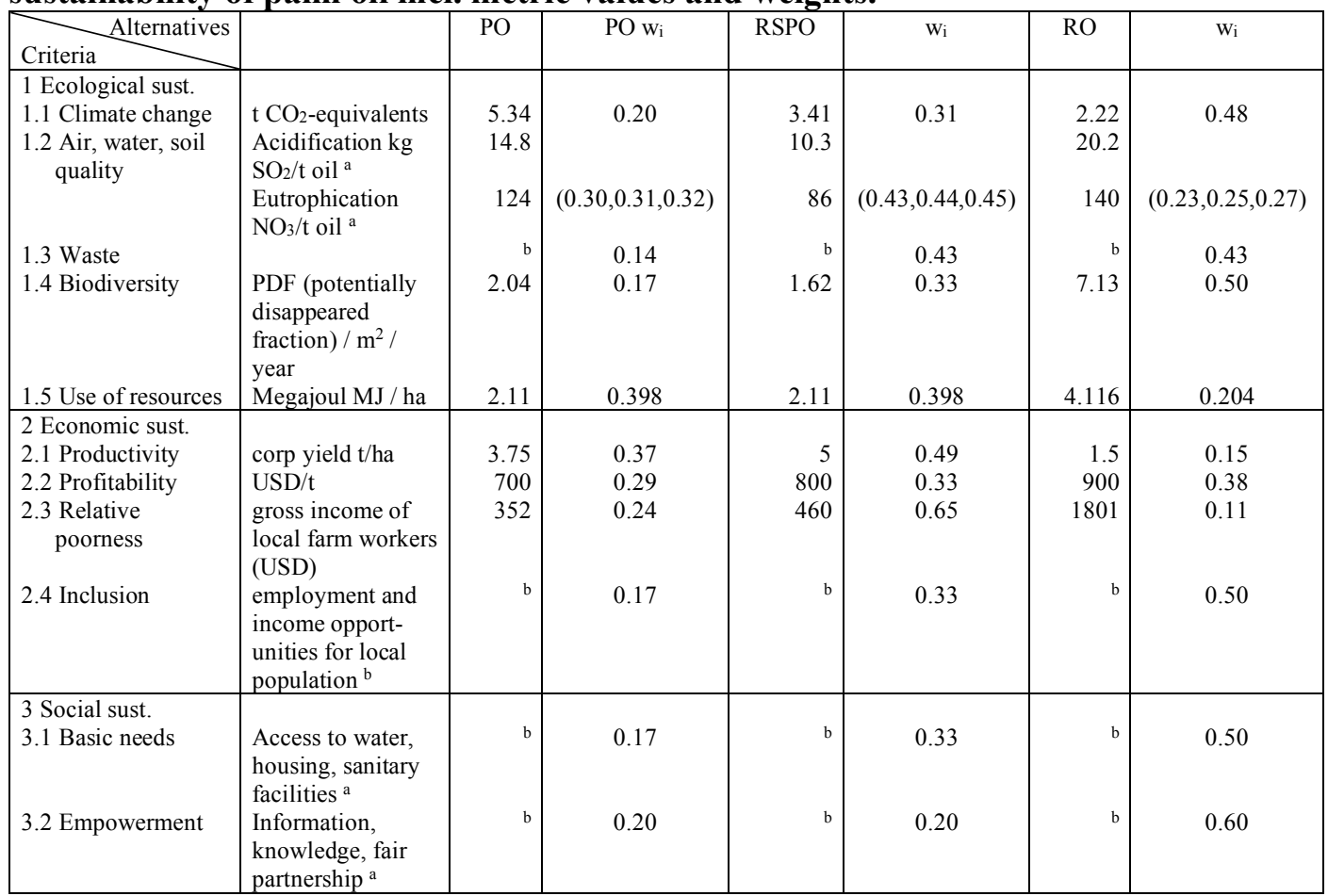

Alternatives $a$ : Conventional palm oil (PO), RSPO-certified palm oil (RSPO), Rapeseed oil (RO)

a Two indicators available, the bandwidth was interpreted as fuzzy numbers.

${ }^{\mathrm{b}}$ As results from literature are ambiguous or not completely comparable, simplified ratings were used.

To approximate the importance of the criteria of the AHP model, we asked eight national and international experts coming from different field of expertise (food producers, agriculture, NGOs, government, science). Unsurprisingly, due to their different professional occupation and varying aims of their organizations, the evaluations based on pairwise comparisons were quite heterogeneous.

Table 2. Absolute weights of experts E1-E8 and transforming them into fuzzy priorities for criteria and sub-criteria.

\begin{tabular}{|l|c|c|c|c|c|c|c|c|c|c|c|c|}
\hline Experts & E1 & E2 & E3 & E4 & E5 & E6 & E7 & E8 & min & mean & max & $\widetilde{W}_{i}$ \\
\hline 1 Ecological sust. & 0.47 & 0.41 & 0.33 & 0.59 & 0.43 & 0.43 & 0.69 & 0.22 & 0.22 & 0.45 & 0.69 & $(0.22,0.45,0.69)$ \\
1.1 Climate change & 0.20 & 0.41 & 0.20 & 0.25 & 0.33 & 0.32 & 0.46 & 0.16 & 0.16 & 0.29 & 0.46 & $(0.16,0.29,0.46)$ \\
1.2 Air, water, soil quality & 0.20 & 0.12 & 0.20 & 0.14 & 0.12 & 0.23 & 0.21 & 0.19 & 0.12 & 0.18 & 0.23 & $(0.12,0.18,0.23)$ \\
1.3 Waste & 0.20 & 0.07 & 0.20 & 0.14 & 0.04 & 0.04 & 0.06 & 0.19 & 0.04 & 0.12 & 0.20 & $(0.04,0.12,0.20)$ \\
1.4 Biodiversity & 0.20 & 0.26 & 0.20 & 0.33 & 0.38 & 0.23 & 0.15 & 0.26 & 0.15 & 0.25 & 0.38 & $(0.15,0.26,0.38)$ \\
1.5 Use of resources & 0.20 & 0.14 & 0.20 & 0.14 & 0.13 & 0.19 & 0.12 & 0.19 & 0.12 & 0.16 & 0.20 & $(0.12,0.16,0.20)$ \\
2 Economic sust. & 0.05 & 0.26 & 0.33 & 0.08 & 0.14 & 0.14 & 0.09 & 0.46 & 0.05 & 0.19 & 0.46 & $(0.05,0.19,0.46)$ \\
2.1 Productivity & 0.07 & 0.07 & 0.30 & 0.08 & 0.17 & 0.09 & 0.15 & 0.24 & 0.07 & 0.15 & 0.30 & $(0.07,0.15,0.30)$ \\
2.2 Profitability & 0.04 & 0.07 & 0.10 & 0.04 & 0.17 & 0.10 & 0.09 & 0.33 & 0.04 & 0.12 & 0.33 & $(0.04,0.12,0.33)$ \\
2.3 Relative poorness & 0.44 & 0.44 & 0.30 & 0.44 & 0.50 & 0.43 & 0.35 & 0.24 & 0.24 & 0.39 & 0.50 & $(0.24,0.39,0.50)$ \\
2.4 Inclusion & 0.44 & 0.42 & 0.30 & 0.44 & 0.17 & 0.38 & 0.41 & 0.19 & 0.17 & 0.34 & 0.44 & $(0.17,0.34,0.44)$ \\
3 Social sust. & 0.47 & 0.33 & 0.33 & 0.33 & 0.43 & 0.43 & 0.22 & 0.32 & 0.22 & 0.36 & 0.47 & $(0.22,0.36,0.47)$ \\
3.1 Basic needs & 0.80 & 0.75 & 0.75 & 0.50 & 0.88 & 0.50 & 0.75 & 0.50 & 0.50 & 0.68 & 0.88 & $(0.50,0.68,0.88)$ \\
3.2 Empowerment & 0.20 & 0.25 & 0.25 & 0.50 & 0.13 & 0.50 & 0.25 & 0.50 & 0.13 & 0.32 & 0.50 & $(0.13,0.32,0.50)$ \\
\hline
\end{tabular}


Combing the AHP criteria weights in Table 2 with the quantitative evaluations of the alternatives in Table 1 finally delivers fuzzy priorities for the three alternatives. The aggregated priority vector for the alternatives $a$ of the AHP hierarchy (Figure 1) amounts to $\widetilde{W}_{a}=\{(0.06,0.22,0.53),(0.10,0.36,0,89),(0.13,0.42,0,97)\}$ compared to $W_{a}=$ $(0.21,0.36,0.43)$ if all data were aggregated confirming the AIJ approach (building geometric means of the pairwise comparisons and combing them with the quantitative data, i.e. crisp numbers, in Table 1).

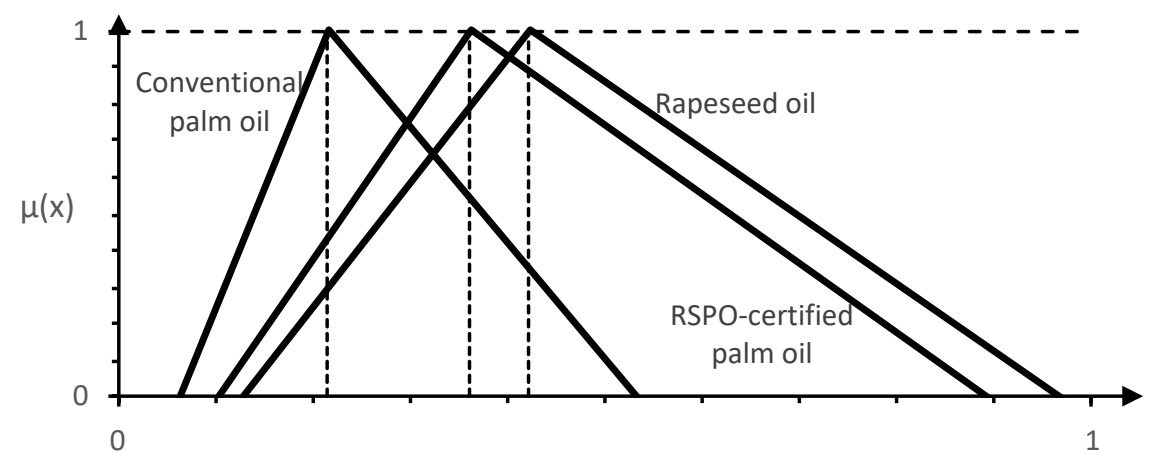

Figure 1. Final weighting for alternatives aggregating individual judgments into fuzzy priorities.

(Remark: All evaluations were consistent with CR < 0.1; (Saaty, 1995))

\section{Conclusions and Limitations}

In the case presented herein, the huge range of the fuzzy priorities shows, that the group decision was by far not homogeneous. There is a good chance that the alternative RSPOcertified palm oil should be evaluated better than the alternative rapeseed oil (using AIJ, rapeseed oil would be definitely evaluated to be the most sustainable alternative). Even the least evaluated alternative conventional palm oil was partly better evaluated than the other two alternatives (even though, the distribution of the fuzzy numbers rather suggests less favorability of conventional palm oil in view of sustainability). Obviously, the assessment of sustainability significantly depends on the individual position of the decision makers and their associated organization. E.g., NGO-representatives weighted environmental criteria considerably higher compared to decision makers from food producers. By implementing our mythology, the diversity of evaluations still is visible, heterogeneitywhich is quite often part of GDM - is not erased. Of course, it depends on the main goal of a GDM process. If the goal is to visualize heterogeneity, our approach seems to be quite beneficial. However, if the goal is to make an actual decision rather than initiating a discussion process, usual approaches following AIJ would be more appropriate. This is a clear limitation of our methodology. Another limitation has to be seen in the fact, that we applied AIP. Only in this case the presented approach delivers valid approximations as for the aggregation of pairwise comparisons the geometric mean has to be used. However, this our methodology could of course be further developed to cover AIJ as well.

\section{References}

Bozdag, C. E., Kahraman, C. and Ruan, D. (2003) 'Fuzzy group decision making for selection among computer integrated manufacturing systems', Computers in Industry, 
ISAHP Article: A Style Guide for Paper Proposals To Be Submitted to the International Symposium on the Analytic Hierarchy Process 2020, Web Conference.

51(1), pp. 13-29. doi: 10.1016/S0166-3615(03)00029-0.

Buchholz, T. et al. (2009) 'Multi Criteria Analysis for bioenergy systems assessments', Energy Policy, 37(2), pp. 484-495. doi: 10.1016/j.enpol.2008.09.054.

Calizaya, A. et al. (2010) 'Multi-criteria decision analysis (MCDA) for integrated water resources management (IWRM) in the Lake Poopo basin, Bolivia', Water Resources Management, 24(10), pp. 2267-2289. doi: 10.1007/s11269-009-9551-x.

Cay, T. and Uyan, M. (2013) 'Evaluation of reallocation criteria in land consolidation studies using the Analytic Hierarchy Process (AHP)', Land Use Policy. Elsevier Ltd, 30(1), pp. 541-548. doi: 10.1016/j.landusepol.2012.04.023.

Chang, C. W., Wu, C. R. and Lin, H. L. (2009) 'Applying fuzzy hierarchy multiple attributes to construct an expert decision making process', Expert Systems with Applications. Elsevier Ltd, 36(4), pp. 7363-7368. doi: 10.1016/j.eswa.2008.09.026. Dong, Y. et al. (2010) 'Consensus models for AHP group decision making under row geometric mean prioritization method', Decision Support Systems. Elsevier B.V., 49(3), pp. 281-289. doi: 10.1016/j.dss.2010.03.003.

Escobar, M. T. and Moreno-Jiménez, J. M. (2007) 'Aggregation of individual preference structures in AHP-group decision making', Group Decision and Negotiation, 16(4), pp. 287-301. doi: 10.1007/s10726-006-9050-x.

Forman, E. and Peniwati, K. (1998) 'Aggregating individual judgments and priorities with the Analytic Hierarchy Process', European Journal of Operational Research, 108(1), pp. 165-169. doi: 10.1016/S0377-2217(97)00244-0.

Van Den Honert, R. C. (1998) 'Stochastic group preference modelling in the multiplicative AHP: A model of group consensus', European Journal of Operational Research, 110(1), pp. 99-111. doi: 10.1016/S0377-2217(97)00243-9.

Kuhlman, T. and Farrington, J. (2010) 'What is sustainability?', Sustainability, pp. 34363448. doi: 10.3390/su2113436.

Ling, X. and Wu, S. (2004) 'Using fuzzy Analytic Hierarchy Process and fuzzy comprehensive evaluation in decision making for satellite mobile communication systems', A Collection of the 22nd AIAA International Communications Satellite Systems Conference and Exhibit, May 9, 2004 - May 12, 2004, 2(May), pp. 847-852.

Saaty, T. (1995) Decision Making for Leaders. The Analytic Hierarchy Process for Decisions in a Complex World. Pittsburgh: RWS Publications.

Schmidt, J. H. (2010) 'Comparative life cycle assessment of rapeseed oil and palm oil', International Journal of Life Cycle Assessment, 15(2), pp. 183-197. doi:

10.1007/s11367-009-0142-0.

Srdjevic, B. (2007) 'Linking analytic hierarchy process and social choice methods to support group decision-making in water management', Decision Support Systems, 42(4), pp. 2261-2273. doi: 10.1016/j.dss.2006.08.001.

Srdjevic, B. et al. (2013) 'A two-phase algorithm for consensus building in AHP-group decision making', Applied Mathematical Modelling. Elsevier Inc., 37(10-11), pp. 66706682. doi: 10.1016/j.apm.2013.01.028.

Srdjevic, B. and Medeiros, Y. D. P. (2008) 'Fuzzy AHP assessment of water management plans', Water Resources Management, 22(7), pp. 877-894. doi: 10.1007/s11269-007-9197-5.

Wi, H. et al. (2009) 'Modeling and analysis of project team formation factors in a project-oriented virtual organization (ProVO)', Expert Systems with Applications, 36(3), pp. 5775-5783. doi: 10.1016/j.eswa.2008.06.116.

Zadeh, L. A. (1965) 'Fuzyy Sets', Information and Control, 8, pp. 338-353. 